\title{
Curved crack patterns by drying bacteria suspensions
}

\author{
Zhengyang Liu, Tianyi Lin, Yiming Qiao, Xiang Cheng, Xiaolei Ma
}

Department of Chemical Engineering and Materials Science, University of Minnesota

\section{Bacteria mobility alters drying crack patterns!}
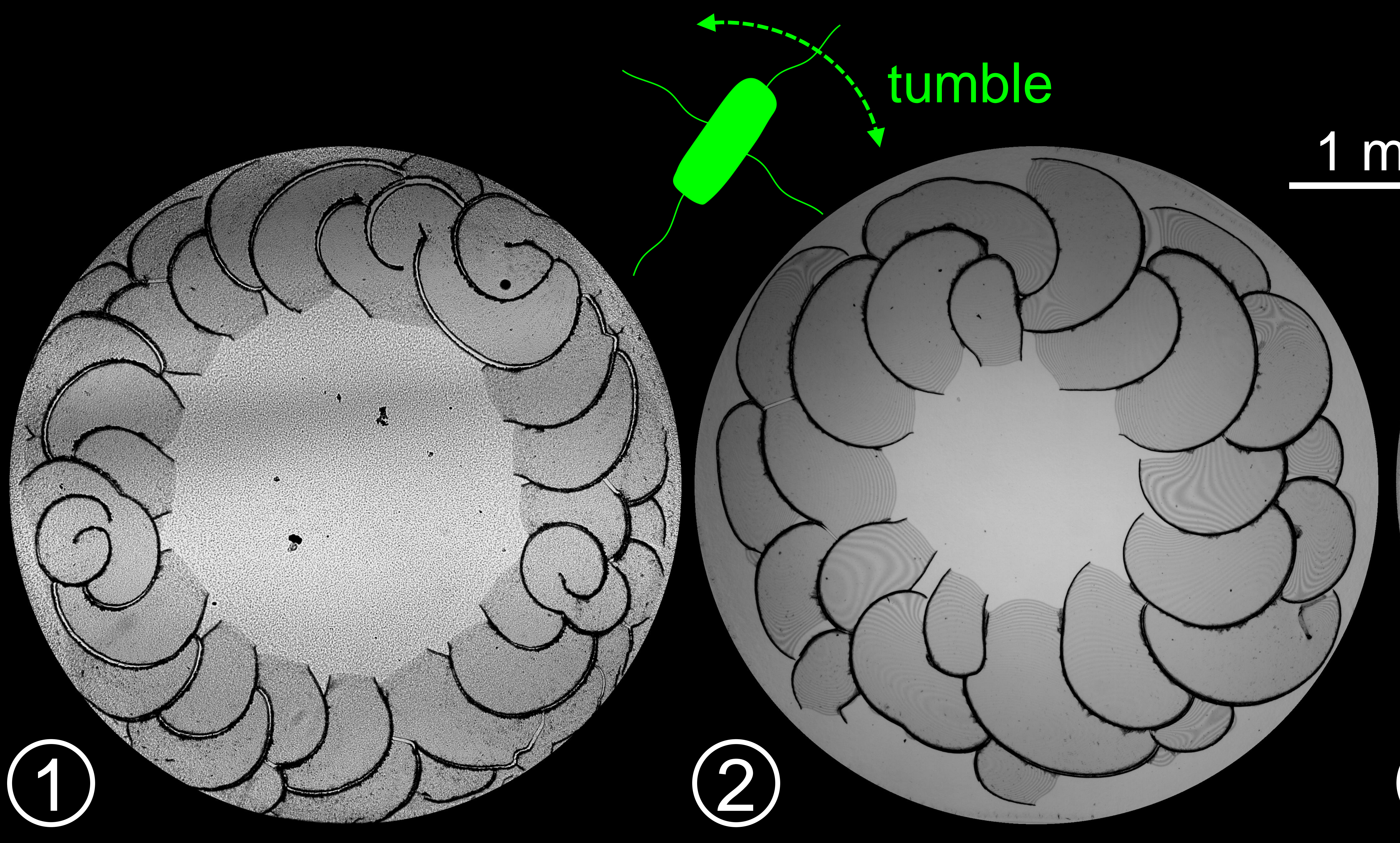

(3)

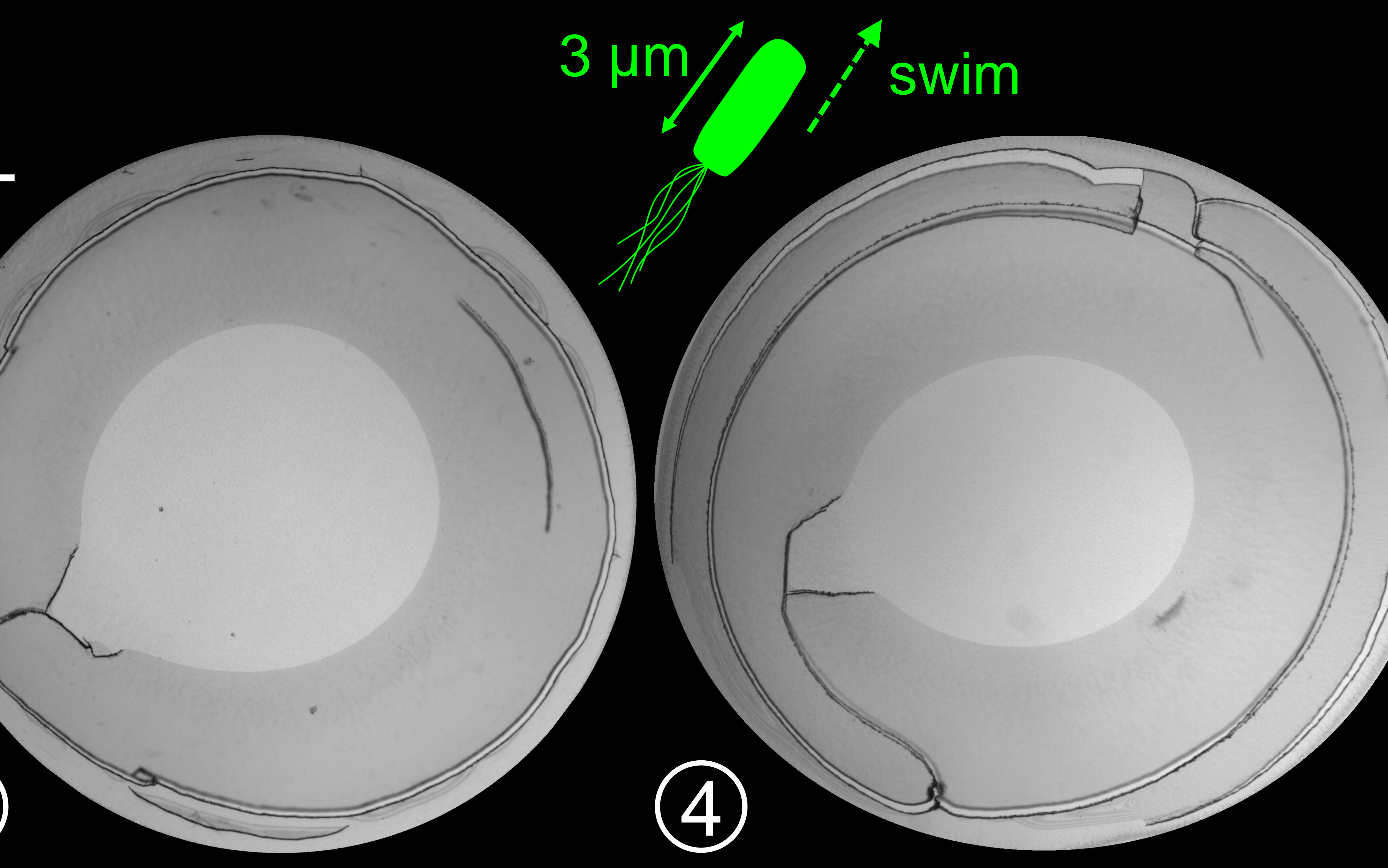

spiral-like cracks in dried E. coli tumbler drops

circular cracks in dried E. coli swimmer drops

Particulate suspensions are prone to crack as the tensile stress in the film builds up when undergoing drying. In contrast to straight cracks in dried colloidal films, two new types curved crack patterns are found by drying drops of bacteria Escherichia coli (E. coll) in water on glass slides. (1, 3: dilute; 2, 4: concentrated)

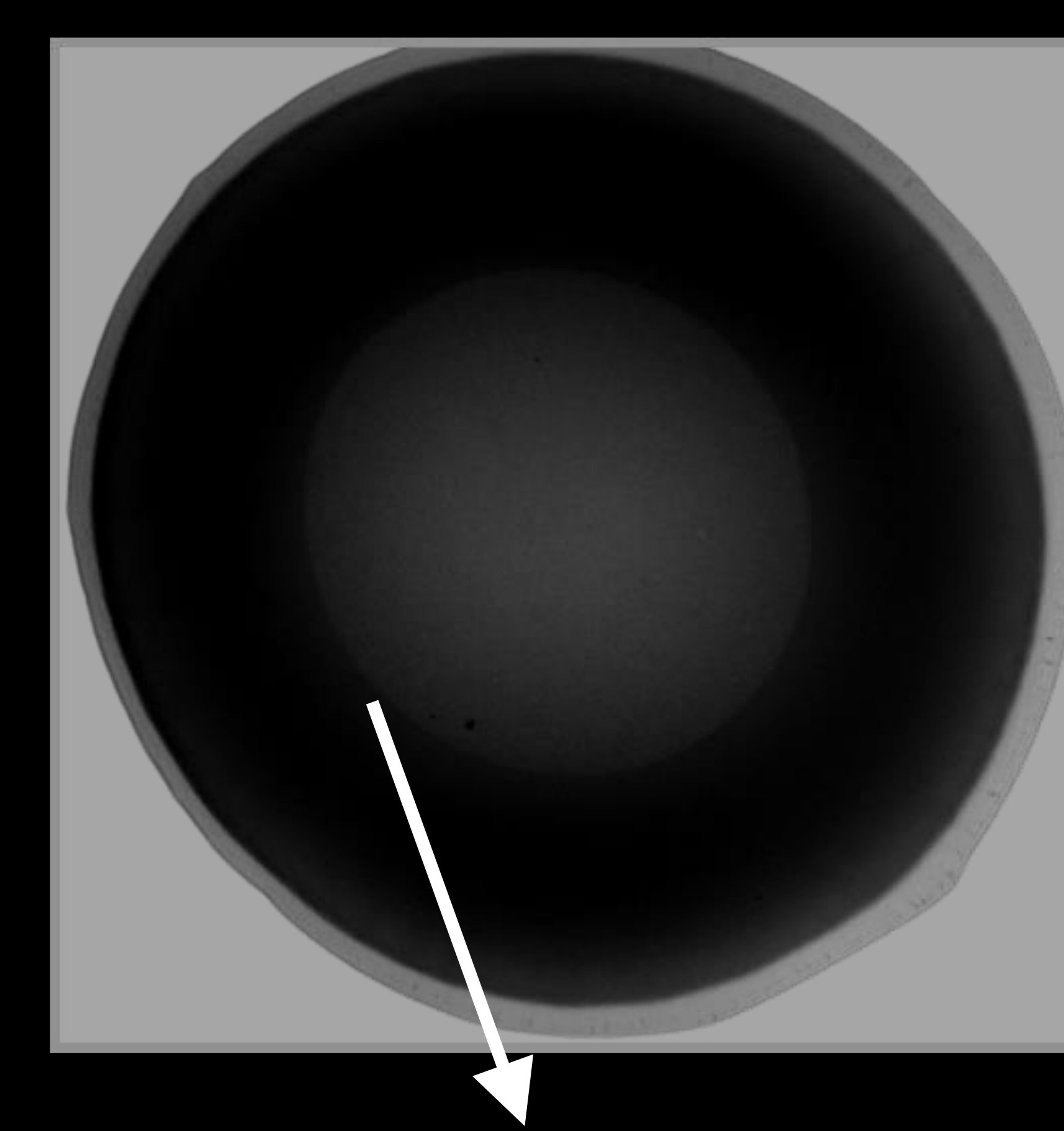

first drying front
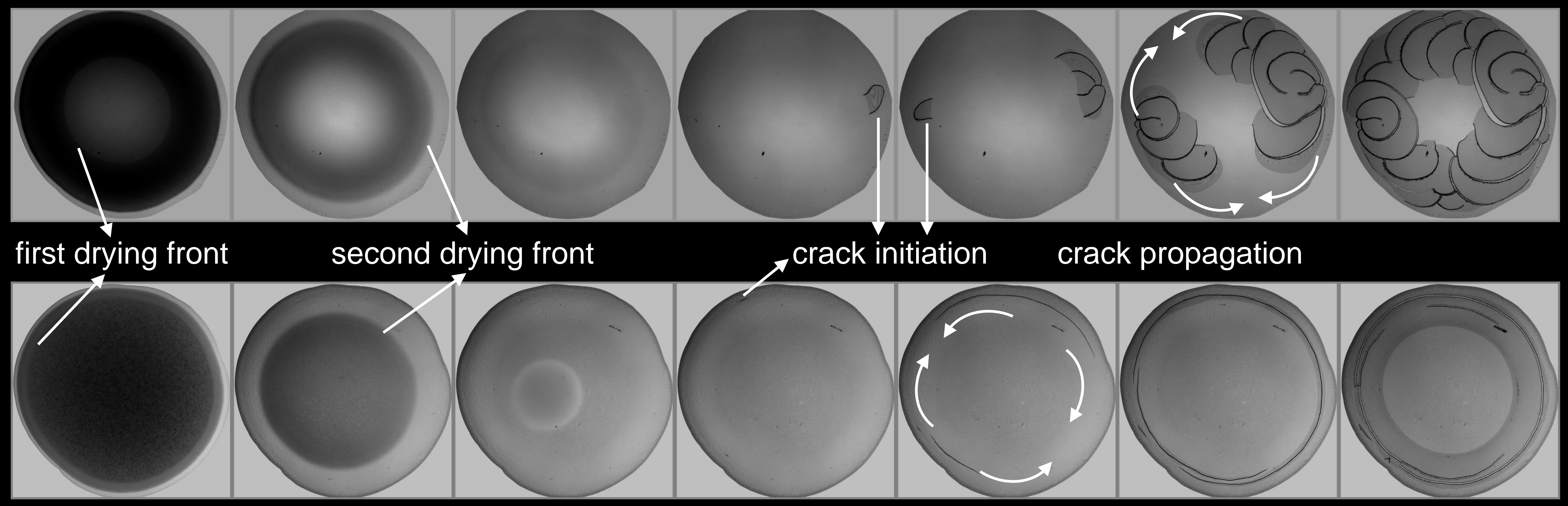

Spiral-like cracks are observed in dried E. coli tumbler drops, whereas dried E. coli swimmer drops display circular cracks. These results suggest bacteria mobility could alter the direction of tensile stress development, leading cracks to propagate in different directions. 